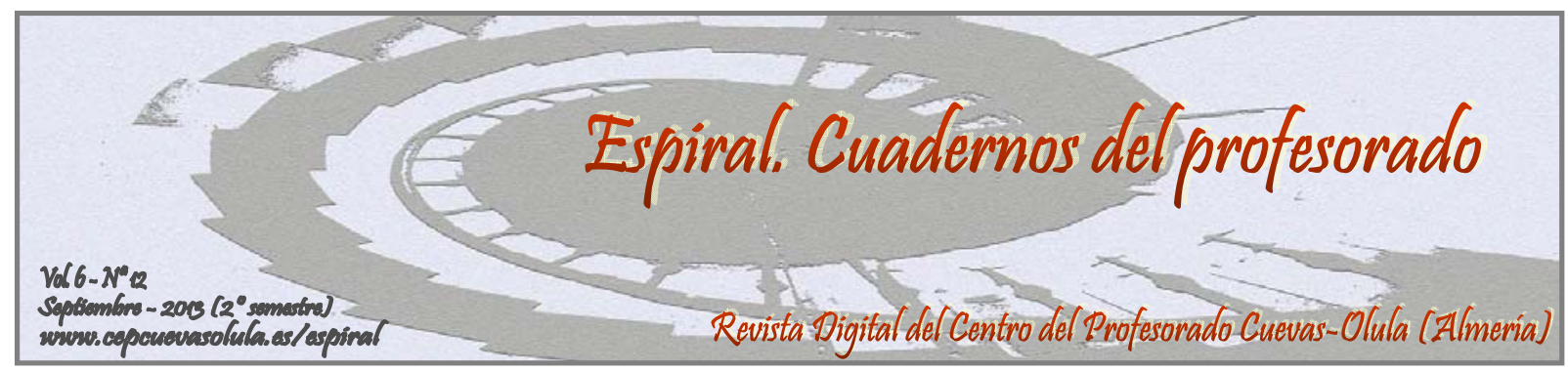

\title{
UNA EXPERIENCIA DE EVALUACIÓN, INVESTIGACIÓN E INNOVACIÓN DESDE UNA PERSPECTIVA DE ASESORAMIENTO COLABORATIVO
}

\author{
AN EVALUATION, RESEARCH AND INNOVATION EXPERIENCE FROM A \\ COLLABORATIVE COUNSELING PERSPECTIVE
}

\section{Carlos Monge López y David Montalvo Saborido}

Facultad de Educación, Universidad de Alcalá (Campus de Guadalajara), Guadalajara, España

RESUMEN: En este artículo se presenta un estudio de caso único cuyo objetivo fundamental fue desarrollar una experiencia de investigación evaluativa mediante la metodología de procesos como recurso del asesoramiento colaborativo. En concreto, desde esta perspectiva se seleccionaron las actividades tutoriales específicas con el alumnado como objeto de mejora. Así, se procuró seguir estas fases cíclicas para la mejora y el cambio: (a) creación de condiciones, (b) revisión general de la situación, (c) clarificación y búsqueda de soluciones, (d) planificación, (e) desarrollo de la innovación y seguimiento del nuevo plan y (f) evaluación y propuestas de mejora. Sin embargo, a pesar de no desarrollarse todas ellas, sí se puede percibir una evidencia sobre los resultados, además de poder compararse con otras investigaciones semejantes. De esta manera, lo más destacado de este estudio fue la colaboración entre la mayor parte de los miembros del centro educativo en cuestión, lo que pretende atribuirse a la metodología de asesoramiento empleada.

Palabras clave: actividades tutoriales, cambio en la escuela, estudio de caso, metodología de procesos.

ABSTRACT: In this paper we present a single case study whose main objective was to develop an evaluative research experience through the process methodology as collaborative counseling resource. Specifically, in this view were selected specific tutorial activities with students for improvement. Thus, we tried to follow these cyclical phases for improvement and change: (a) creating conditions, (b) general review of the situation, (c) clarification and solutions, (d) planning, (e) development of innovation and following the new plan and (f) evaluation and improvement proposals. However, despite not develop all of them, we can perceive an evidence of the results as well as to compare with other similar investigations. In this way, the highlight of this study was the collaboration between the majority of the members of the school in question, which we attributed to the methodology employed.

Key words: Case study, change in schools, process methodology, tutorial activities.

Monge López, C., y Montalvo Saborido, D. (2013). Una experiencia de evaluación, investigación e innovación desde una perspectiva de asesoramiento colaborativo. Espiral. Cuadernos del Profesorado, 6(12), 51-60. Disponible en: http://www.cepcuevasolula.es/espiral.

Fecha de recepción: 28/04/2013

Fecha de aceptación: 12/09/2013
Enviar correspondencia a: carlos.monge@edu.uah.es 


\section{1.- INTRODUCCIÓN}

El presente texto muestra una experiencia de evaluación, investigación e innovación en un centro educativo concreto. Así, se aplicó una investigación evaluativa (plasmada en el Plan de Evaluación) que partió desde la metodología de procesos propia del asesoramiento colaborativo. Y, finalmente, se propusieron mejoras reflejadas en un Proyecto de Innovación Educativa.

El objetivo primordial del Plan de Evaluación fue diseñar una propuesta de innovación centrada en la realización de un plan de investigación evaluativa sobre el programa de tutorías de un centro educativo, al cual aplicar una innovación desde una perspectiva de asesoramiento colaborativo. Es decir, el Plan de Evaluación fue el preámbulo del Proyecto de Innovación Educativa que le debía seguir, fue la justificación, la base, las raíces de ese proyecto. A partir de la evaluación del programa de tutorías (en concreto las actividades tutoriales específicas con el alumnado) se elaboraron propuestas de mejora que serían concretadas en la innovación. Pero qué es la evaluación en sí misma. Pues ésta es un proceso de recogida de información y de reflexión sistemática, integral e integrada, y rigurosa cuya finalidad es la comprensión crítica de una institución con objeto de mejorarla (Gather, 2004).

Aunque actualmente, siguiendo la legislación vigente, se denominan "medidas de actuación tutoriales” incluidas en el Proyecto Educativo de centro, aquí, para facilitar su comprensión, se nombra como Plan de Acción Tutorial.

Concretamente, las actividades específicas de tutoría con el alumnado se fundamentan desde estas tres perspectivas: (a) la orientación psicopedagógica, (b) la orientación formativa-profesional y (c) la educación en valores. Pero no son vistas todas ellas como entes separados e independientes, sino que forman un conjunto mediante la interconexión.

Por otro lado, tal y como dicta el Plan de Acción Tutorial, los principios que lo rigen son: (a) personalización de la enseñanza (que supone, a su vez, individualización, integración e inclusión), (b) concepto de educación como algo más que instruir y (c) educación integral. Como señala el Plan de Acción Tutorial, "la tutoría es una actividad inherente a la función del profesor que se realiza individual y colectivamente, con la finalidad de facilitar la integración personal y los procesos de aprendizaje".

Desde el marco legislativo, cabe señalar que:

- La acción tutorial orientará el proceso educativo individual y grupal del alumnado en el conjunto de la etapa.

- El tutor o tutora coordinará la intervención educativa del conjunto del profesorado y mantendrá una relación continuada y sistemática con la familia.

- El equipo directivo garantizará, con carácter general, que la persona responsable de la tutoría imparta docencia al grupo de alumnos en, al menos, tres áreas del currículo.

- El tutor, con el asesoramiento del responsable de orientación del centro, incluirá, dentro del horario semanal en el que permanece con el grupo, actividades de seguimiento y orientación del proceso de enseñanza y actividades que contribuyan al desarrollo de las habilidades propias de la competencia social y ciudadana, de la competencia para aprender a aprender, de la competencia de autonomía e iniciativa personal y de la competencia emocional.

- El centro podrá programar modelos alternativos de tutoría que garanticen una acción individualizada y continua con el alumnado y su familia.

- El centro docente programará actividades que faciliten la transición del alumnado de Educación Infantil a Educación Primaria, y de ésta a la Educación Secundaria Obligatoria.

Desde el punto de vista legislativo, la Ley Orgánica 2/2006, de 3 de mayo, de Educación propone una educación fundamentada en una serie de valores.

La educación primaria contribuirá a desarrollar en los niños y niñas las capacidades que les permitan: conocer y apreciar los valores y las normas de convivencia, aprender a obrar de acuerdo con ellas, prepararse para el ejercicio activo de la ciudadanía y respetar los derechos humanos, así como el 
pluralismo propio de una sociedad democrática (Ley Orgánica de Educación, Título I, Capítulo II, Artículo 17).

Y, precisamente, esta idea defendida en el marco legal, al igual que en el teórico, es un pilar sobre los que se sustentan esas tutorías.

Desde el plano teórico, el Plan de Acción Tutorial defiende que el desarrollo de la función tutorial puede contribuir a:

- Hacer efectivos principios y fines de la educación.

- El cumplimiento de objetivos de las etapas educativas de infantil y primaria.

- La calidad de la enseñanza.

- La mejora y regulación de los procesos de enseñanza-aprendizaje.

- Orientar al alumnado en su proceso de enseñanza-aprendizaje y en otros ámbitos, como el personal, familiar, social y comunitario.

- El desarrollo de la capacidad para tomar decisiones (desarrollo de la identidad y autonomía personal).

El Plan de Evaluación que aquí concierne se centra en lo que el propio Plan de Acción Tutorial denomina como "actividades tutoriales específicas con los alumnos". Esto se dio, entre otras causas, a que, tras varias entrevistas con la orientadora de un centro educativo concreto, se percibió que el profesorado realizaba una serie de demandas, lo que conllevó a planificar una serie de actividades tutoriales específicas con el alumnado.

Pero, tras todo ello, ¿para qué evaluar? Pues bien, la evaluación pretende comprender y transformar la actividad educativa del Plan de Acción Tutorial centrado en las actividades específicas mencionadas antes, así como transformar en el sentido de mejorar (cambiar). El cambio en la organización, tal y como lo definen Miles y Ekholm (1986), es un esfuerzo sistemático dirigido a cambiar las condiciones de aprendizaje y otras condiciones internas relacionadas con el propósito de lograr las metas educativas. O bien, el cambio en la organización también puede ser un proceso a lo largo del cual la organización en su conjunto y cada uno de sus miembros han de aprender nuevos modos de pensar y actuar (Fullan, 1990).

A modo de síntesis, el Plan de Evaluación surgió como un intento para mejorar algunos aspectos fundamentales del Plan de Acción Tutorial de un centro educativo en concreto.

Por otro lado, si hasta aquí se introducen los conceptos de investigación y de evaluación, ahora se procede a presentar el término "innovación educativa”. Ésta se puede definir como el “conjunto de ideas, procesos y estrategias, más o menos sistematizados, mediante las cuales se trata de introducir y provocar cambios en las prácticas educativas vigentes" (Carbonell, 2002, p. 11). Otra forma de conceptualizar la innovación educativa puede ser la formulada por Rivas: "la innovación educativa es la incorporación de algo nuevo en el sistema de la institución escolar, cuyo resultado es la modificación de la estructura y operaciones, de tal modo que mejoren sus efectos en orden al logro de los objetivos" (2000, p. 27). Desde un punto de vista más didáctico, se entiende por innovación educativa a los "cambios institucionalizados concebidos y realizados en el seno de los centros educativos” (Gairín, 2009, p. 23).

La innovación aquí presentada se puede enmarcar atendiendo a las clasificaciones establecidas por Rivas (2000) y Moschen (2008). Así, por un lado y considerando la perspectiva de Rivas (2000), esta innovación puede percibirse como:

- Según los componentes: (a) una operación para el mantenimiento de los límites, (b) cuyo tamaño y extensión concierne a un centro educativo completo, (c) con redistribuciones del tiempo y del espacio, (d) con la modificación e implementación de nuevos objetivos instructivo-formativos, (e) donde se redefinen los roles, (f) así como los valores, las concepciones y creencias, (g) que parte de la modificación de la estructura y relaciones de las partes, (h) que promueve nuevos métodos de socialización en el centro, (i) donde se procura fomentar la conexión con el contexto y (j) cuyos resultados cambian los procedimientos didácticos, organizativos y curriculares, materiales y, finalmente, de agrupamientos. 
- Según el modo de realización: (a) adición, (b) reforzamiento, (c) alteración y (d) reestructuración.

- Según la amplitud: facultativas o parciales.

- Según la intensidad: adicionales.

- Y, por otra parte, considerando las nuevas tipologías que añade Moschen (2008), puede clasificarse de la siguiente manera:

- Según los costes: económicamente bajos, pero considerables en esfuerzo y dedicación.

- Según el tiempo: corto plazo (2-3 años).

- Según la participación y gestión institucional: parcialmente asistida.

Por último, puede considerarse que, independientemente del Proyecto de Innovación Educativa, ya sólo esta experiencia de asesoramiento desde la metodología de procesos constituye una innovación para este centro educativo.

\section{2.- METODOLOGÍA}

\section{Material y método}

En primer lugar, cabe señalar que se trata de un estudio de caso único. Concretamente, la investigación evaluativa se enfoca en un Centro de Educación Infantil y Primaria de una localidad con unos 5.000 habitantes, de naturaleza eminentemente agrícola e industrial, relativamente próxima a la capital de provincia y con 348 alumnos y 32 docentes.

En segundo lugar, se describe el modelo de evaluación seguido. De esta manera, el punto de vista desde el que seguir el presente plan de evaluación es una síntesis de estas tres perspectivas (González, 2003): técnica, cultural y política.

La primera de ellas concibe la organización como una entidad real, externamente observable. Lo cual supone una epistemología objetivista, donde conocer es reproducir aquello a lo que se puede acceder mediante la observación directa. Además, estudia preferentemente el sistema, con sus estructuras, funciones, conductas, productos... Se preocupa por el orden y el consenso. Para producir conocimiento recurre al análisis y explicación estructural y funcional (sistémico), a los diseños experimentales o cuasi-experimentales, a las formas cuantitativas de recoger y analizar la información y a la representación numérica de datos siguiendo la lógica matemática. Y responde a un interés normativo, es decir, técnico.

La perspectiva cultural concibe la organización como realidad cultural internamente construida. Lo cual presupone una epistemología subjetivista, donde conocer es reinventar aquello a lo que se puede acceder por observación participante. Estudia preferentemente los significados, las creencias, los valores, la cultura... También, se preocupa por cómo se manifiesta subjetiva o internamente, asumiendo que la realidad organizativa se caracteriza por la ambigüedad y la adaptación. Para producir conocimiento recurre al análisis fenomenológico (cognitivo, simbólico), a diseños biográficos y etnográficos, a formas cualitativas de recoger y analizar información y a la representación discursiva de datos siguiendo la lógica narrativa.

La perspectiva política concibe la organización como realidad política externamente construida, lo cual presupone una epistemología constructivista (social) relativista, donde conocer es representar aquello a lo que se puede acceder por observación directa y participante. Estudia preferentemente las ideologías, intereses, metas, poder, etc. Se preocupa por el porqué y para qué, asumiendo que la realidad organizativa se caracteriza por el dominio, el conflicto y la negociación. Para producir conocimiento recurre al análisis crítico (dialéctico), a los estudios de casos, a las formas cualitativas de recoger y analizar, a la representación discursiva y a las descripciones críticas atentas a dimensiones éticas y políticas.

El modelo de evaluación de este plan sigue unos aspectos propios, recogidos de distintos modelos de evaluación. Es decir, al igual que sucede con las perspectivas, la evaluación sigue un modelo sintetizado de otros muchos modelos, donde se procura recoger los elementos más interesantes de cada uno de ellos para aplicar en esta evaluación. Así, siguiendo la clasificación propuesta por 
Miguel (1989), los modelos de evaluación que más se aproximan a ésta son: los que postulan criterios mixtos o integradores (donde se tiene en cuenta tanto los resultados como los procesos) y los que se centran en la evaluación para la mejora. Pero, con carácter general, se tienen en cuenta todos los grandes grupos que él propone.

Desde el paradigma cualitativo, se tiene en cuenta el modelo de Tyler (1942) (consecución de objetivos), así como el de Cronbach (1963) (la evaluación que se usa para mejorar un programa mientras éste se está aplicando, lo que contribuye más al desarrollo de la educación que la evaluación usada para estimar el valor del producto de un programa ya concluido), el de Scriven (1967) (con la evaluación sumativa para aquel proceso orientado a comprobar la eficacia del programa y tomar decisiones sobre su continuidad), el de Stake (1967) (puesto que combina descripción y valoración, con datos y percepciones de los participantes), etc.

En resumen, el Plan de Evaluación que aquí concierne intenta enfocarse hacia un modelo integrador, donde se combina lo objetivo con lo subjetivo y lo constructivo, lo externo con lo interno, lo cualitativo con lo cuantitativo, lo normativo con lo prescriptivo, etc. La evaluación pretende compendiar los aspectos más positivos de cada perspectiva. Pero no sólo eso, la evaluación sigue una adaptación del modelo de procesos. La evaluación aquí está enmarcada en un perspectiva de asesoramiento colaborativo, donde una persona externa al centro moviliza a parte de la comunidad educativa en la medida de lo posible para establecer una colaboración mutua (Armengol, 2002; Carpio y Guerra, 2008; Domingo, 2005; Torrego, 2008).

Además, esta evaluación es de carácter formal, ya que se procura que sea sistemática, fundamentada, rigurosa y ética. Pero, aunque la evaluación no tenga un carácter informal, sí se han de contemplar las informalidades puesto que, además de un medio más para la recogida de información, son espacios y tiempos donde se da mayor libertad para la expresión personal.

Por otro lado, los términos operativos seguidos en el Plan de Evaluación hacen referencia a: los objetivos perseguidos, los criterios de evaluación, los estándares marcados y, finalmente, los instrumentos para la recogida de datos. De esta manera, los términos operativos giran en torno a los siguientes objetivos:

- Analizar el documento donde se recoge la acción tutorial del centro. Aquí, los criterios de evaluación hacen referencia a: (a) el tiempo, (b) el espacio, (c) el personal implicado, (d) los recursos materiales, (e) la metodología, (f) los objetivos perseguidos y (g) la evaluación. Para ello, se aplica el análisis de documentos y la observación directa.

- Observar la práctica de la acción tutorial. En este caso, los criterios de evaluación se formulan sobre: (a) la rentabilidad del tiempo y (b) la satisfacción hacia las actividades propuestas en el Plan de Acción Tutorial. Y, para ello, se utilizaron encuestas y análisis de documentos.

- Analizar las posibles mejoras. Aquí, los criterios de evaluación se centran en: (a) la formulación individual de propuestas de mejora y (b) la colaboración para las mismas. Para ello se recurrió al análisis de documentos y a las entrevistas en profundidad.

Por último, la metodología empleada para promover el cambio dentro de la organización ha sido la metodología de procesos, adaptándola a las características del contexto educativo. Así, siguiendo este modelo, las fases para emprender la evaluación y la innovación son las siguientes (Armengol, 2002; Domingo, 2005; Torrego, 2008):

- Creación de condiciones para poder abordar la cuestión de la acción tutorial.

- Revisión general de la situación de la acción tutorial en el centro.

- Clarificación y búsqueda de soluciones.

- Planificación de la acción tutorial en el centro.

- Desarrollo de la innovación y seguimiento del nuevo plan.

- Evaluación y propuestas de mejora.

Concretamente, en este caso de metodología, se sigue siempre un proceso que parte desde la primera fase, que debe ser siempre mantenida durante toda la experiencia de investigación evaluativa e 
innovación. Posteriormente se pasa, una a una, a las siguientes fases. De esta manera, el Proyecto de Innovación Educativa, como resultado del Plan de Evaluación, fue integrado en un proceso cíclico en referente a la práctica docente, donde se desarrollaron estas tres fases: evaluación, formación e innovación. Además, las fases del proceso de innovación (diseño, adopción, implantación e institucionalización) fueron, también, de carácter cíclico.

\section{3.- RESULTADOS}

Los datos y la información obtenida se estructuran según las fases de la metodología de procesos (Armengol, 2002; Domingo, 2005; Torrego, 2008).

Para la creación de condiciones, la primera actuación fue insertar aquí una reflexión específica sobre la relevancia del tema en el centro educativo, tratando de, tal y como dicta el modelo de procesos, enraizar el plan en la historia del centro y concretar específicamente la respuesta que se daría con esta metodología a la acción tutorial. Desde la colaboración, se propuso como necesidad principal las actividades tutoriales específicas con el alumnado. Esta necesidad ha sido propuesta por el conjunto del Claustro, tras debatir que la mayoría de los docentes no están satisfechos con tales actividades. Y para evitar que indirectamente se produjera un proceso de asunción directa de la mayoría de los compromisos por parte del grupo interno (constituido por los coordinadores del proyecto en el centro y el equipo directivo), se han adoptado medidas de distribución de responsabilidades. Por último, se han establecido unos tiempos y espacios específicos para el cumplimiento de las responsabilidades y compromisos asumidos, tanto individual como grupalmente.

La segunda fase del modelo de procesos (revisión general de la situación de la acción tutorial en el centro) fue realizada colaborativamente desde dentro del centro pero con apoyo externo (realizando éste el Plan de Evaluación). Así, los resultados más destacados indicaron:

- Respecto al número de tutorías trimestrales, el estándar propuesto es de una tutoría directa con los alumnos cada trimestre, y la realidad del centro fue que realizan una cada mes. Y la duración de las mismas se correspondió con el estándar (50 minutos por sesión).

- Los espacios destinados para las mismas propuestos en los estándares son: los docentes, los recreativos y los de circulación y comunicación. En cambio, el Plan de Acción Tutorial sólo contempla a los docentes y los recreativos. Además, el estándar señala una flexibilidad en cuanto a los espacios, permitiendo la salida de los mismos, pero, tras el análisis de documentos, esto no se plantea.

- El número de personas que se considera idóneo en el Plan de Evaluación para impartir cada tutoría específica con el alumnado es tres. Una se corresponde con el tutor de cada grupo-clase, otra con la orientadora y otra con alguien externo al centro (de la Administración, de las familias, expertos externos, etc.). Sin embargo, el documento referido a tales efectos solamente recoge la participación directa del tutor y de la orientadora.

- Uno de los varios indicadores se refiere al número de ciclos a los que van dirigidas las actividades tutoriales específicas con el alumnado, indicando el estándar que han de ser para todos los ciclos que se imparten en el centro. De hecho, la planificación del centro va más allá y dirige tales actividades para todos los cursos.

- Un dato significativo es que no se contemplan los recursos materiales referentes a las Tecnologías de la Información y Comunicación.

- Respecto a la metodología, ésta ha de ser flexible, abierta y adaptable y promover el trabajo cooperativo. Estos estándares se cumplen ampliamente en estas actividades tutoriales.

- Los objetivos, tal y como marcan los estándares, han de contribuir a la consecución de todos los objetivos generales de cada etapa propuestos por la legislación vigente, así como de los objetivos marcados para cada área, formularse en torno a contenidos interdisciplinares, a todos los temas transversales y a todas la competencias básicas:

- Los objetivos señalados por la legislación para la etapa de Educación Infantil y que el Plan de Acción Tutorial no contempla son los siguientes: (a) iniciarse en las habilidades lógico-matemáticas, en la lecto-escritura y en el movimiento, el gesto 
y el ritmo; y (b) desarrollar habilidades comunicativas en diferentes lenguajes y formas de expresión. Y los de Educación Primaria que deja aislados tal documento son: (a) conocer y utilizar de manera apropiada la lengua castellana y desarrollar hábitos de lectura; (b) adquirir en, al menos, una lengua extranjera la competencia comunicativa básica que les permita expresar y comprender mensajes sencillos y desenvolverse en situaciones cotidianas; (c) fomentar la educación vial y actitudes de respeto que incidan en la prevención de los accidentes de tráfico; (d) iniciarse en la utilización, para el aprendizaje, de las tecnologías de la información y la comunicación desarrollando un espíritu crítico ante los mensajes que reciben y elaboran; (e) utilizar diferentes representaciones y expresiones artísticas e iniciarse en la construcción de propuestas visuales; y (f) desarrollar las competencias matemáticas básicas e iniciarse en la resolución de problemas que requieran la realización de operaciones elementales de cálculo, conocimientos geométricos y estimaciones, así como ser capaces de aplicarlos a las situaciones de su vida cotidiana.

- Todos los objetivos de cada área de Educación Infantil son contemplados en las actividades específicas. Y en Educación Primaria no se contemplan los objetivos más técnicos propios de cada área.

- Aunque las competencias básicas sí se contemplan en los objetivos del Plan de Acción Tutorial para Educación Primaria, posteriormente no se concretan mediante las actividades tutoriales específicas con el alumnado. De hecho, aquellas competencias básicas que no se desarrollan en estas actividades son: (a) competencia en comunicación lingüística, (b) competencia matemática, (c) competencia cultural y artística y (d) tratamiento de la información y competencia digital. Y los temas transversales que no están presentes en los objetivos son el de las Nuevas Tecnologías y el de la educación vial.

- De interdisciplinariedad hay que señalar que sí se alcanzan los estándares propuestos.

- Respecto a la evaluación, cabría mencionar que, tal y como plantean los estándares, sí se formulan procedimientos y criterios de evaluación para el alumnado, así como una evaluación para el propio plan. Lo que no plantea es una evaluación de la propia evaluación (metaevaluación).

- Los estándares marcan que los minutos de máximo aprovechamiento de cada sesión han de ser 40 de los 50 minutos que dura cada una. Estos datos se han obtenido realizando una aproximación a la media aritmética de una sesión por cada grupo-clase (salvo en Educación Infantil por inviabilidad). De entre esos 15 minutos no aprovechados al máximo están 5 por retraso del personal implicado, 3 de castigos (sanciones e interrupciones), 5 en traslados y dos en que los niños se organicen.

- El profesorado, contrariamente al estándar propuesto, sostuvo que el nivel evaluado es adecuado. Los alumnos lo evaluaron como muy positivo. El servicio de orientación y el equipo directivo lo hicieron como adecuado. Y, respecto a las familias, el centro no posee información al respecto, ya que, tal y como señala la memoria del servicio de orientación, "los alumnos no han hecho comentario alguno en el centro escolar sobre los mismos, salvo cuando se les ha preguntado de forma directa, siendo un escaso número de familias, los que han comentado con sus hijos los temas ahí expuestos”.

- Pasando ahora al criterio de formulación de propuestas de mejora, los estándares mínimos contemplan la participación y formulación por parte de los miembros del centro (alumnos, docentes, personal de orientación y equipo directivo), de la Administración y de las familias. En cambio, el Plan de Acción Tutorial sólo contempla la de los docentes, la orientadora, el equipo directivo y las familias; en realidad, tras varias entrevistas informales, hasta la fecha siempre las ha realizado la persona encargada de proponer los documentos base que han de fomentar los procesos reflexivos sobre la acción tutorial (la orientadora). 
La tercera y cuarta fase se culminan con la elaboración de un Proyecto de Innovación Educativa. En él se revisaron y modificaron las actividades del Plan de Acción Tutorial, y se crearon unas actividades nuevas para poder solventar las necesidades encontradas. De esta manera, los dos objetivos principales fueron: (a) presentar la innovación como un cambio en la mentalidad de toda la comunidad educativa del centro que tienda hacia la mejora colectiva, (b) adentrar al centro en el campo de la investigación para que sus propios miembros sean capaces de promover una mejora en otro momento ante una nueva necesidad planteada. Por otro lado, se englobaron todos los contenidos en torno a tres ejes: (a) enseñar a convivir y aprender a construir la igualdad entre hombre y mujeres, (b) enseñar a ser persona y a emprender y (c) enseñar a pensar. También, se puede considerar como elementos importantes a los principios a seguir en el proyecto de innovación, tales como:

- La investigación interdisciplinar para la reconstrucción del conocimiento como eje del proceso de innovación.

- La práctica misma que legitima la innovación educativa.

- La formación que constituye la esencia de las innovaciones educativas para la transformación cultural que procuran la mejora del nivel de vida individual y social.

- La autonomía para que se generan los procesos de innovación educativa.

- Finalmente, las actividades propuestas como innovadoras fueron las siguientes:

- Actos culturales solidarios.

- Introducción al aprendizaje colaborativo.

- "Aprendiendo a conducir”.

- "La mesa de comunicación”.

- “Tiempo de concordia”.

- Dinámicas para la confianza, motivación y participación del profesorado.

- "La naturaleza dentro del centro".

Por último, tanto la puesta en práctica de la innovación (quita fase) como su evaluación y propuestas de mejora (sexta fase), todavía no se han llevado a la práctica. Sin embargo, la Comisión de Coordinación Pedagógica ya está trabajando para su aplicación en el próximo curso académico.

En resumen, la utilización de la metodología de procesos empleada en este estudio de caso promueve la colaboración entre la mayor parte de los miembros del centro educativo en cuestión, lo que origina unas propuestas innovadoras centradas en mejorar algunos de los procesos educativos.

\section{4.- REPERCUSIONES SOBRE LA COMUNIDAD EDUCATIVA}

Con carácter general, se puede señalar que la experiencia de investigación, evaluación e innovación aquí analizada facilita los procesos de colaboración entre algunos de los integrantes del centro educativo presentado, y cuyo fin último es producir una mejorar de aquellos aspectos educativos que considerar deficientes.

También, el presente estudio de caso único contiene una amplia semejanza con una de las investigaciones posiblemente más relevantes al respecto. Si bien es cierto que Torrego (2008) muestra una temática diferente (la mejora de la convivencia), en general las conclusiones finales sí pueden considerarse como estrechamente semejantes. Así, en ambos estudios se señala que la metodología de procesos en el asesoramiento colaborativo puede ser un recurso favorable para la mejora de algunos elementos de los centros educativos.

Por otro lado, la investigación aquí presentada puede ser llevada a cabo en múltiples contextos. Por ejemplo, mientras se estaba llevando a cabo la presente experiencia, se producía una similar en otro de los centros educativos de la localidad. Concretamente, se trata de un Instituto de Educación Secundaria Obligatoria con el que comparten un Proyecto de Innovación Educativa, así como una larga trayectoria de coordinación entre estos centros. Por ende, una de las posibles investigaciones futuras puede ser llevar a cabo otra experiencia de investigación evaluativa desde la metodología de procesos con el fin de: (a) comparar los procesos de asesoramiento colaborativo entre Centros de Educación Infantil y Primaria con los Institutos de Educación Secundaria, (b) analizar con 
mayor profundidad la eficiencia de la metodología de procesos como facilitadora de la mejora educativa y (c) poder compartir una experiencia que abarque un mayor contexto.

Fundamentalmente, las repercusiones sobre la comunidad educativa se centran sobre dos elementos: (a) la mejora de las actividades tutoriales específicas y (b) la formación docente. En el primero ellos, el modelo seguido repercute directamente sobre la organización y planificación de los documentos internos del centro y, especialmente, sobre el alumnado. Y, paralelamente, la propia metodología de procesos facilita que el profesorado se desarrolle como investigador de su práctica educativa. Además, todo ello incide sobre la calidad de la escuela, animando al conjunto de la comunidad educativa a cambiar sus perspectivas y participar en los procesos de innovación.

A modo de síntesis, puede mencionarse que el asesoramiento colaborativo y la utilización de la metodología de procesos son unos recursos que pretenden mejorar algunos procesos educativos mediante la colaboración entre docentes. Además, puede ser entendido como un proceso formativo hacia la investigación, la evaluación y la innovación en educación.

\section{5.- CONCLUSIONES}

A modo de conclusión final, tras los resultados obtenidos, puede señalarse que el asesoramiento constituye una herramienta fundamental para facilitar los procesos de cambio y mejora en los centros educativos. Concretamente, desde la perspectiva del asesoramiento colaborativo es posible considerar que la metodología de procesos sea un recurso fundamental para mejorar los procesos educativos de los centros (Carpio y Guerra, 2008; Domingo, 2005; Armengol, 2002; Torrego, 2008).

Respecto a los objetivos perseguidos, el principal es proponer una innovación educativa a partir del Plan de Evaluación empleado desde la metodología de procesos. Y, para ello, se sigue una serie de pasos: (a) análisis del documento donde se recoge la acción tutorial del centro, (b) observación de la práctica de la acción tutorial y (c) análisis de las posibles mejoras.

En relación con las dificultades encontradas en esta investigación, puede señalarse que la propuesta de innovación educativa todavía no ha sido llevada a la práctica. Sin embargo, sí existe una consistencia empírica para poder concluir que esta metodología de asesoramiento facilita la colaboración entre la mayor parte de los miembros del centro educativo estudiado.

Se puede concluir que si las instituciones colaboran en procesos de asesoramiento (como por ejemplo: universidad-escuela, centros de formación-escuelas, etc.) se producen tanto innovaciones como recursos formativos del profesorado. Concretamente, los centros educativos son entornos donde conviven diversas personas, como los docentes, por lo que es posible considerar como elemental la coordinación y colaboración entre ellos. Y esto se puede llevar a cabo con el asesoramiento colaborativo, especialmente con la metodología de procesos.

\section{6.- REFERENCIAS}

Armengol, C. (2002). El trabajo en equipo en los centros educativos. Barcelona: Cisspraxis.

Carbonell, J. (2002). El profesorado y la innovación educativa. En: P. Cañal (Coord.), La innovación educativa (pp. 11-27). Madrid: Universidad Internacional de Andalucía-Ediciones Akal.

Carpio, A. y Guerra, L. M. (2008). Una experiencia de asesoramiento colaborativo como estrategia de apoyo a los profesores para la implementación de un programa de tutorías universitarias. Profesorado. Revista de Currículum y Formación del Profesorado [en línea], 12(1). Disponible en: http://www.ugr.es/ recfpro/rev121COL12.pdf [consultado el 11 de septiembre de 2013].

Cronbach, L. J. (1963). Course improvement through evaluation. Teachers College Record, 64(5), 72-86.

Domingo, J. (2005). Las prácticas de asesoramiento a centros educativos: una revisión del modelo de proceso. Archivos Analíticos de Políticas Educativas [en línea], 13(17). Disponible en: http://epaa.asu.edu/ojs/article/view/122/248 [consultado el 11 de septiembre de 2013].

España. Ley Orgánica 2/2006, de 3 de mayo, de Educación. Boletín Oficial del Estado, 4 de mayo de 2006, núm. 106, pp. 17158-17207. 
Fullan, M. D. (1990). Changing school culture through staff development. Alejandría: Association for Supervision and Curriculum Develeopment Yearbook.

Gairín, J. (2009). Cambio y mejora. La innovación en el aula, equipo de profesores y centro. En: J. Paredes y A. de la Herrán (Coords.), La práctica de la innovación educativa (pp. 21-48). Madrid: Editorial Síntesis.

Gather, M. (2004). La evaluación de las instituciones no se mide: se construye, se negocia, se practica y se vive. En: M. Crahay (Coord.), Problemática y metodología de la evaluación de las instituciones educativas (pp. 203-224). Bruselas: De Boeck.

González, M. T. (Coord.) (2003). Organización y gestión de centros escolares: dimensiones y procesos. Madrid: Pearson Educación.

Miguel, M. de (1989). Modelos de investigación sobre organizaciones educativas. Revista de Investigación Educativa, 7(13), 21-56.

Miles, M. B. y Ekholm, M. (1986). What is School Improvement? En: W. G. Van Venzel (Coord.), Making School Improvement Work: A Conceptual Guide to Practice (pp. 33-67). Leuven: ACCO.

Moschen, J. C. (2008). Innovación educativa. Decisión y búsqueda permanente (segunda edición). Buenos Aires: Bonum.

Rivas, M. (2000). Innovación educativa: teoría, procesos y estrategias. Madrid: Editorial Síntesis.

Scriven, M. (1967). The methodology of evaluation. Chicago: Rand McNally.

Stake, R. (1967). The countenance of educational evaluation. Teachers College Record, 68(4), pp. 23-40.

Torrego, J. C. (2008). Un estudio sobre la utilización de la metodología de procesos como estrategia de formación del profesorado en relación con la mejora de la convivencia. Profesorado. Revista del Currículum y Formación del Profesorado [en línea], 12(1). Disponible en: http//:www.ugr.es /local/recfpro/rev121ART13.pdf [consulta: 2011, 23 de noviembre].

Tyler, R. W. (1942). Basic principles of curriculum and instructions. Chicago: Universidad de Chicago.

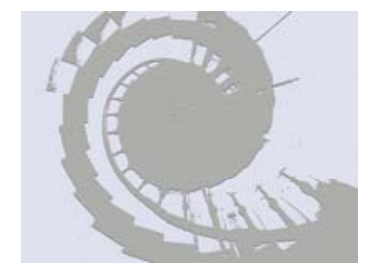

